\title{
Psychotherapy via Telehealth during the COVID-19 Pandemic in Australia-Experience of Clients with a Diagnosis of Borderline Personality Disorder
}

\author{
Nitin P Dharwadkar ${ }^{1,3}$, Jillian H Broadbear ${ }^{2,3}$, Parvaneh Heidari ${ }^{2,3}$, Lukas Cheney ${ }^{2}$ \& Sathya Rao ${ }^{2,3}$ \\ ${ }^{1}$ Department of Psychiatry, Central Clinical School, Monash University, Melbourne, Victoria, Australia \\ ${ }^{2}$ Spectrum Service for Personality Disorder, Richmond, Victoria, Australia \\ ${ }^{3}$ Faculty of Medicine, Nursing and Health Sciences, Monash University, Clayton, Victoria, Australia \\ Correspondence: Dr Parvaneh Heidari, Spectrum Service for Personality Disorder, Level 2, 110 Church St, \\ Richmond, Victoria, 3121, Australia. Tel: (+613)-84-13-8750. E-mail: parvaneh.heidari@easternhealth.org.au
}

Received: October 21, 2021 Accepted: November 22, 2021 Online Published: November 24, 2021

doi:10.5539/gjhs.v14n1p29 URL: https://doi.org/10.5539/gjhs.v14n1p29

\begin{abstract}
Objective: To investigate the transition to, and experience of, telehealth in people with a diagnosis of borderline personality disorder (BPD).

Method: A cross-sectional study using an online survey was conducted in a specialist clinic for personality disorders in March-May 2020.

Results: Thirty-seven clients (48\% response rate) completed the survey. Two participants (5.4\%) were decided not to receive treatment via telehealth. Transitioning from in-person to telehealth, the majority of participants had few or no technical issues (51.4\%). Telephone, video-conferencing and a mix of telephone and video-conferencing were used. Positive and negative experiences were endorsed asking about the effectiveness of telehealth. While some participants were whether unsure $(32 \%)$ or not $(19 \%)$ interested in telehealth following pandemic, half acknowledged the presence of telehealth (54.8\%) and wanted to have the option of telehealth following pandemic $(48.6 \%)$.
\end{abstract}

Conclusions: Despite some shortcomings associated with telehealth, almost every client continued to attend appointments and half of the study participants wanted to have the option of telehealth in the future. Healthcare policymakers and mental health managers should consider the challenges described in this study while developing telehealth guidelines to best support people experiencing problems living with the psychiatric diagnoses of BPD.

Keywords: Borderline personality disorder, telehealth, telemental health, COVID-19

\section{Introduction}

On 30 January 2020, the coronavirus disease 2019 (COVID-19) was declared a global public health emergency (World Health Organization, 2020). Its contagious nature and potentially severe outcomes necessitated rapid adaptations across economies, societies, and healthcare systems. Many countries, including Australia, adopted national workplace and community restrictions to limit the spread of the virus. In Australia, in-person clinical appointments were replaced wherever possible with telehealth services (Mikakos, 2020). Telehealth is defined as the provision of healthcare remotely by means of telecommunications technology (World Health Organization, 2010). Although there is evidence for the efficiency, cost-effectiveness, and utility of mental health services delivered via telehealth (Langarizadeh et al., 2017), when it comes to borderline personality disorder (BPD), very few studies were available to provide guidance prior to the pandemic (Ben-Zeev, 2014; Kocsis \& Yellowlees, 2018). BPD is the highest prevalence personality disorder, experienced by $1-2 \%$ of the population at some time during their lives (Grant et al., 2008). According to the Diagnostic and Statistical Manual (DSM 5), people with this diagnosis may experience intense, uncontrollable emotions, have trouble with their relationships, find it hard to feel comfortable in themselves, be very impulsive and appear to lead chaotic lives, or intentionally harm themselves as a way of coping.

Prior to 2020, telehealth in Australia was utilized primarily for people residing in rural and remote areas (Bradford et al., 2016). During the COVID-19 restrictions, telehealth medical services were introduced throughout Australia, 
enabling people to access healthcare services from their homes while decreasing the risk of exposure and spread of COVID-19 (Kidd \& Hunt, 2020). The process and funding arrangements for the expansion of tele-mental health services were quickly developed with widespread adoption across the community (Australian Government, 2020).

A meta-analysis that included 2,648 veterans previously reported similar effectiveness in treatment outcomes using both telehealth (video-conferencing and telephone) and in-person psychotherapies. Participants in the meta-analysis had a variety of mental health conditions, mostly trauma, depression, and anxiety (McClellan et al., 2021). Another meta-analysis examined the effectiveness of video-conferencing in the delivery of mental health services. The study concluded that while more rigorous studies are needed to reach a definitive answer, current literature suggests that video-conferencing does not impede clinical outcomes (Batastini et al., 2020). In terms of the acceptability of telehealth, a retrospective study evaluating health record data from two outpatient mental health clinics in the United States (US) that transitioned to telehealth within two days, reported a decrease in scheduled appointments of $10.6 \%$ immediately post transition, increasing by $17.8 \%$ six months post transition (Mishkind et al., 2021).

For many clients and providers, the transition to telehealth services took place over such a short space of time that opportunities for planning and training were minimal. Although some pilot studies investigating the utility of telehealth for the provision of mental health services have been published (Inchausti et al., 2020; Tullio et al., 2020), the efficacy and utility of psychotherapy via telehealth delivery for people diagnosed with BPD are currently unknown. This study aimed to investigate the transition to and experience of telehealth in clients previously receiving in-person psychotherapy for the treatment of BPD.

\section{Method}

A cross-sectional study utilizing an online survey was conducted within a public outpatient specialist personality disorder clinic which treats clients diagnosed with severe and complex personality disorder presentations. The clinic offers a range of evidence-based psychotherapy programs, including dialectical behavioral therapy, mentalization-based therapy, and a 'common factors' treatment program. Clients who were receiving treatment services were invited to participate ( $\mathrm{n}=77$ ) during the first wave of social isolation restrictions (March-May 2020). All clients of the service were aged 18 years or older and diagnosed with BPD according to the Diagnostic and Statistical Manual of Mental Disorders (5th Edition, American Psychiatric Association, 2013). Clients were invited to participate via an email message explaining the purpose of the study with a link to the online survey. The survey contained three sections: 1. Demographic information; 2. Experience of transition to and use of telehealth with questions regarding how telehealth affected their relationship with their clinician and their own mental health, and 3. Pros and cons of telehealth treatment and future use of telehealth. The data were analyzed using descriptive statistics in Microsoft Excel 2010.

Ethics approval was obtained from the institutional ethics committee (LR20/037).

\section{Results}

Thirty-seven clients (48.1\% response rate) completed the survey. Most participants were female ( $86.5 \%)$, aged less than 38 years $(64.8 \%)$, living with family members $(56.8 \%)$, and in the first year of their treatment in the clinic (78.3\%) (see supplementary material). A minority of participants $(n=13,35.1 \%)$ had used telehealth previously for health-related services. Two participants $(5.4 \%)$ had decided not to continue their treatment via telehealth.

Transitioning from in-person to telehealth treatment, the majority of participants had few or no technical issues $(\mathrm{n}=18$ of $35,51.4 \%)$, or only experienced issues initially; however, they either solved the technical issues enabling them to use the videoconference platform or received telehealth by telephone $(n=14,40.0 \%)$. A few had ongoing technical problems $(\mathrm{n}=3,8.5 \%)$. Difficulties were related to the internet (i.e., quality of connection, cost, and equipment).

Telephone $(\mathrm{n}=8,25.8 \%)$, video-conferencing $(\mathrm{n}=9,29.0 \%)$, and mix of telephone and video-conferencing $(\mathrm{n}=14$, $45.1 \%$ ) were used (out of 31 respondents). The most commonly stated reasons for only using the telephone were participants' ongoing unresolved technical issues with video-conference, feeling self-conscious, anxious, or distracted by their own appearance during the video-conference. Participants who were using video-conferencing $(n=24)$ also reported the occasional feeling of insecurity $(n=14,58.3 \%)$ self-consciousness, anxiety $(n=16,66.6 \%)$, or distraction ( $\mathrm{n}=17,70.8 \%)$.

When asked about the effectiveness of telehealth as a platform for receiving mental health services, positive and negative experiences were endorsed (Tables 1 and 2). While more than half of the participants appreciated not having to travel to their appointments, two noted the importance of travel for treatment effectiveness. Table 1 illustrates how some participants felt more relaxed, able to speak more freely, and felt that they made similar 
progress as when they met with their clinician in person. Some found it a bit harder to make progress and more challenging to stay engaged, experiencing lower mood, a worsening of mood fluctuations, or feelings of abandonment. Of clients who reported worsening feelings of abandonment, four reported reliable access to telehealth, two had technical issues, and three only had access to telehealth treatment by phone.

Out of 30 respondents, many participants endorsed that difficult conversations with their clinicians were easier $(\mathrm{n}=9,30 \%)$ or no different $(\mathrm{n}=11,36.7 \%)$ to manage via telehealth than when meeting in person. It was also acknowledged that misunderstandings were more likely when using telehealth $(\mathrm{n}=7,23.3 \%)$, more challenging to resolve $(n=4,13.3 \%)$, and it was easier to exit the conversation $(n=6,20.0 \%)$. Avoiding difficult conversations due to privacy concerns was also endorsed $(\mathrm{n}=4,13.3 \%)$.

Table 1. Experience of receiving psychological treatment via telehealth for people with BPD during COVID-19 restrictions $(\mathrm{N}=35)$

\begin{tabular}{lc}
\hline & N (\%) \\
\hline I like not having the stress of travelling & $20(57.1)$ \\
It is a bit harder to make progress compared with face-to-face but it is working OK & $14(40.0)$ \\
It is challenging to stay engaged when our main communication is via telehealth; it is not working so well as & $13(37.1)$ \\
face-to-face & $10(28.6)$ \\
I feel more relaxed and able to talk more freely & $10(28.6)$ \\
I find that we accomplish a similar amount in sessions as we did prior to using telehealth & $7(20.0)$ \\
I feel a worsening of my feelings of abandonment & $7(20.0)$ \\
I feel that my mood fluctuates more easily, making it more difficult to regulate my emotional responses & $7(20.0)$ \\
My mood feels lower since my treatment sessions moved to telehealth
\end{tabular}

Table 2. Perceived impact of telehealth on treatment of people with BPD during COVID-19 restrictions

\begin{tabular}{|c|c|}
\hline Impact of telehealth & $\mathbf{N}(\%)$ \\
\hline \multicolumn{2}{|l|}{ Communication with the clinician, $\mathrm{n}=29$} \\
\hline Caused some instability in my relationship with my clinician. & $6(20.7)$ \\
\hline Led to improvement in my relationship with my clinician. & $5(17.2)$ \\
\hline Difficult to say whether using telehealth has changed my relationship with my clinician. & $18(62.1)$ \\
\hline \multicolumn{2}{|l|}{ Expressing thoughts about self-harm or suicide, $n=31$} \\
\hline It does not seem to happen more frequently with telehealth & $18(58.0)$ \\
\hline It does seem to happen more frequently when using telehealth & $4(12.9)$ \\
\hline Unsure - it is hard for me to know & $9(29.0)$ \\
\hline \multicolumn{2}{|l|}{ More difficult to control impulses for self-harm?, $n=31$} \\
\hline Always & $1(3.2)$ \\
\hline Usually & $2(6.4)$ \\
\hline Sometimes & $4(12.9)$ \\
\hline Rarely & $12(38.7)$ \\
\hline Never & $12(38.7)$ \\
\hline \multicolumn{2}{|l|}{ More difficult to control feelings of anger?, $n=31$} \\
\hline Always & $0(0.0)$ \\
\hline Usually & $4(12.9)$ \\
\hline Sometimes & $5(16.1)$ \\
\hline Rarely & $10(32.2)$ \\
\hline Never & $12(38.7)$ \\
\hline
\end{tabular}


Harder to establish and maintain agreed treatment boundaries with their clinician?, n=27

\begin{tabular}{|c|c|}
\hline Always & $1(3.7)$ \\
\hline Usually & $1(3.7)$ \\
\hline Sometimes & $4(14.8)$ \\
\hline Rarely & $5(18.5)$ \\
\hline Never & $16(59.2)$ \\
\hline \multicolumn{2}{|c|}{ More likely to experience fatigue when using telehealth?, $n=31$} \\
\hline Always & $1(3.2)$ \\
\hline Usually & $5(16.1)$ \\
\hline Sometimes & $8(25.8)$ \\
\hline Rarely & $6(19.3)$ \\
\hline Never & $11(35.5)$ \\
\hline \multicolumn{2}{|c|}{ Accessibility of individual clinician during the restrictions compared to in-person consultation?, $\mathrm{n}=31$} \\
\hline More accessible & $3(9.6)$ \\
\hline About the same & $24(77.4)$ \\
\hline Less accessible & $4(12.9)$ \\
\hline \multicolumn{2}{|c|}{$\begin{array}{l}\text { Are there aspects of telehealth that work better for you compared with when you received face-to-face } \\
\text { psychological treatment?, } n=31\end{array}$} \\
\hline Yes & $17(54.8)$ \\
\hline No & $9(29.0)$ \\
\hline Unsure & $5(16.1)$ \\
\hline \multicolumn{2}{|c|}{ Would you like to have the option of receiving psychological treatment via telehealth in the future?, $\mathrm{n}=37$} \\
\hline Yes & $18(48.6)$ \\
\hline No & $7(18.9)$ \\
\hline Unsure & $12(32.4)$ \\
\hline
\end{tabular}

\section{Discussion}

This study investigated the transition to and experience of receiving psychotherapy via telehealth from the perspective of clients who have a diagnosis of BPD. Although the transition was rapid, the majority of clients transitioned to telehealth smoothly, with only two clients deciding not to use the telehealth service and three clients having ongoing technical problems using telehealth. Transition difficulties relating to the internet and technology were found in the current and US studies (Mishkind et al., 2021). In the US study, although there was a slight decrease in the number of appointments made immediately after transition - similar to the current study - this number had increased relative to pre-virtualisation six months after transition (Mishkind et al., 2021), suggesting the acceptance of telehealth as a health service tool.

Although the widespread introduction of telehealth has occurred due to the pandemic, its continued use is highly likely post-pandemic. While some participants in the current study were unsure (32\%) or not (19\%) interested in telehealth following the pandemic, half acknowledged the importance of receiving treatment by telehealth during social restrictions and wanted to have the option of telehealth following the pandemic. A survey of mental health clinicians and organizations in the US reported a high likelihood that telehealth use would continue post-pandemic (Molfenter et al., 2021).

Difficult topics and challenging conversations are fundamental to psychotherapy when client presentations include complex trauma, self-harming behavior, suicidality, and interpersonal difficulties (Brodsky \& Stanley, 2013). When asked about managing difficult conversations via telehealth, most in this study found such conversations easier or no different from in-person sessions. The majority reported no change in their likelihood of expressing thoughts regarding self-harm or suicidality. Some participants avoided complex topics when interacting with their clinician via telehealth, citing an increased possibility of misunderstandings and greater difficulty achieving resolution, and sometimes leaving a telehealth session if it became too difficult. This could conceivably slow 
clinical progress, however, there are early indications that treatment engagement via telehealth of people who have serious mental illness may not differ from other forms of mental health service engagement (Miu et al., 2020). Indeed this service conducting this study noted a substantial rise in client engagement compared with previous levels of attendance at in-person sessions (Broadbear et al., 2021).

With respect to symptom expression, close to a quarter of participants perceived that telehealth made it more difficult to control feelings of anger or impulses to self-harm, highlighting the need for guidance in managing risk when conducting treatment via telehealth (Hames et al., 2020; Inchausti et al., 2020; Jobes et al., 2020). It is notable that the service did not experience an increase in serious adverse events during the months that telehealth was the only treatment option.

\subsection{Limitations}

This study was conducted within a specialist personality disorder service which therefore limits generalisation of these findings to the wider population of people with a diagnosis of BPD. Data were collected using an online survey with a cross-sectional design during the first national lockdown in Australia. Longer-term utility of telehealth for people with a diagnosis of BPD is yet to be reported. Survey questions did not differentiate between individual and group therapy. However, as almost all participants were receiving individual therapy (transition of group sessions to the virtual format took substantially longer), it is assumed that questions mainly reflected participants' experience of the individual sessions.

\section{Conclusion}

Results of this study describe the challenges and benefits of telehealth from the perspective of clients of a specialist service for personality disorder. Healthcare policymakers and mental health managers should consider the challenges outlined in this study when developing telehealth guidelines for people with a diagnosis of BPD. Despite some shortcomings associated with telehealth, almost every client continued to attend appointments. Participants did not report harm from the delivery of psychotherapy via telehealth, suggesting that telehealth need not be contraindicated as a treatment option. Participant responses also indicate that using telehealth to deliver psychotherapy may be acceptable in the post-COVID world too. This is particularly important given the 'rolling' nature of the pandemic-related restrictions into the foreseeable future.

\section{Competing Interests Statement}

The authors declare that there are no competing or potential conflicts of interest.

\section{References}

American Psychiatric Association. (2013). Diagnostic and statistical manual of mental disorders (DSM-5®) (5th ed.): Diagnostic and statistical manual of mental disorders. Am Psychiatric Assoc, 21. https://doi.org/10.1176/appi.books.9780890425596

Australian Government. (2020). COVID-19 Temporary Medicare Benefits Schedule Telehealth Services, Mental Health Services. Australian Government from http://www.mbsonline.gov.au/internet/mbsonline/publishing.nsf/Content/0C514FB8C9FBBEC7CA25852E 00223AFE/\$File/COVID-19\%20Temporary\%20MBS\%20telehealth\%20Services\%20-\%20Mental\%20Heal th\%2008052020.pdf

Batastini, A. B., Paprzycki, P., Jones, A. C., \& MacLean, N. (2020). Are video-conferenced mental and behavioral health services just as good as in-person? A meta-analysis of a fast-growing practice. Clinical Psychology Review, 101944. https://doi.org/10.1016/j.cpr.2020.101944

Ben-Zeev, D. (2014). Technology-based interventions for psychiatric illnesses: improving care, one patient at a time. Epidemiology and psychiatric sciences, 23(4), 317-321. https://doi.org/10.1017/S2045796014000432

Bradford, N. K., Caffery, L. J., \& Smith, A. C. (2016). Telehealth services in rural and remote Australia: a systematic review of models of care and factors influencing success and sustainability. Rural and remote health, 16(4), 4268. https://doi.org/10.22605/RRH3808

Broadbear, J. H., Heidari, P., Dharwadkar, N. P., Cheney, L., \& Rao, S. (2021). Telehealth Psychotherapy for Severe Personality Disorder during COVID-19: Experience of Australian Clinicians. Global Journal of Health Science, 13(12). https://doi.org/10.5539/gjhs.v13n12p61

Brodsky, B. S., \& Stanley, B. (2013). The dialectical behavior therapy primer: How DBT can inform clinical practice. John Wiley \& Sons. https://doi.org/10.1111/bjop.12061

Grant, B. F., Chou, S. P., Goldstein, R. B., Huang, B., Stinson, F. S., Saha, T. D., ... \& Pickering, R. P. (2008). 
Prevalence, correlates, disability, and comorbidity of DSM-IV borderline personality disorder: results from the Wave 2 National Epidemiologic Survey on Alcohol and Related Conditions. The Journal of Clinical Psychiatry, 69(4), 533-545.

Hames, J. L., Bell, D. J., Perez-Lima, L. M., Holm-Denoma, J. M., Rooney, T., Charles, N. E., . . Fondacaro, K. M. (2020). Navigating uncharted waters: Considerations for training clinics in the rapid transition to telepsychology and telesupervision during COVID-19. Journal of Psychotherapy Integration, 30(2), 348. https://doi.org/10.1037/int0000224

Inchausti, F., MacBeth, A., Hasson-Ohayon, I., \& Dimaggio, G. (2020). Telepsychotherapy in the age of COVID-19: A commentary. https://doi.org/10.1037/int0000222

Jobes, D. A., Crumlish, J. A., \& Evans, A. D. (2020). The COVID-19 pandemic and treating suicidal risk: The telepsychotherapy use of CAMS. Journal of Psychotherapy Integration, 30(2), 226. https://doi.org/10.1037/int0000208

Kidd, M., \& Hunt, G. (2020). COVID-19: Whole of population telehealth for patients, general practice, primary care and other medical services. Retrieved from https://www.health.gov.au/ministers/the-hon-greg-hunt-mp/media/covid-19-whole-of-population-telehealthfor-patients-general-practice-primary-care-and-other-medical-services

Kocsis, B. J., \& Yellowlees, P. (2018). Telepsychotherapy and the therapeutic relationship: Principles, advantages, and case examples. Telemedicine and e-Health, 24(5), 329-334. https://doi.org/10.1089/tmj.2017.0088

Langarizadeh, M., Tabatabaei, M. S., Tavakol, K., Naghipour, M., Rostami, A., \& Moghbeli, F. (2017). Telemental health care, an effective alternative to conventional mental care: A systematic review. Acta Informatica Medica, 25(4), 240. https://doi.org/10.5455/aim.2017.25.240-246

McClellan, M. J., Osbaldiston, R., Wu, R., Yeager, R., Monroe, A. D., McQueen, T., \& Dunlap, M. H. (2021). The effectiveness of telepsychology with veterans: A meta-analysis of services delivered by video-conference and phone. Psychological Services. https://doi.org/10.1037/ser0000522

Mikakos, J. (2020). Pandemic plan for the Victorian Health Sector. Melbourne, Australia: Victorian State Government. Retrieved from https://www2.health.vic.gov.au/about/publications/researchandreports/covid19-pandemic-plan-for-vic

Mishkind, M. C., Shore, J. H., Bishop, K., D'Amato, K., Brame, A., Thomas, M., \& Schneck, C. D. (2021). Rapid conversion to telemental health services in response to COVID-19: experiences of two outpatient mental health clinics. Telemedicine and e-Health, 27(7), 778-784. https://doi.org/10.1089/tmj.2020.0304

Miu, A. S., Vo, H. T., Palka, J. M., Glowacki, C. R., \& Robinson, R. J. (2020). Teletherapy with serious mental illness populations during COVID-19: telehealth conversion and engagement. Counselling Psychology Quarterly, 1-18. https://doi.org/10.1080/09515070.2020.1791800

Molfenter, T., Heitkamp, T., Murphy, A. A., Tapscott, S., Behlman, S., \& Cody, O. J. (2021). Use of Telehealth in Mental Health (MH) Services During and After COVID-19. Community Mental Health Journal, 1-8. https://doi.org/10.1007/s10597-021-00861-2

Tullio, V., Perrone, G., Bilotta, C., Lanzarone, A., \& Argo, A. (2020). Psychological support and psychotherapy via digital devices in Covid-19 emergency time: Some critical issues. Medico-Legal Journal, 0025817220926942. https://doi.org/10.1177/0025817220926942

World Health Organization. (2010). Telemedicine: opportunities and developments in member states. Report on the second global survey on eHealth. Geneva: World Health Organization

World Health Organization. (2020). Rolling updates on coronavirus disease (COVID-19). Retrieved from https://www.who.int/emergencies/diseases/novel-coronavirus-2019/events-as-they-happen 


\section{Supplementary material}

Demographic characteristics of people with a diagnosis of BPD who received treatment via telehealth during COVID-19 restrictions

\begin{tabular}{|c|c|}
\hline Characteristics & $\mathbf{N}(\%)$ \\
\hline \multicolumn{2}{|l|}{ Gender, $n=37$} \\
\hline Female & $32(86.5)$ \\
\hline Male & $4(10.8)$ \\
\hline Not stated & $1(2.7)$ \\
\hline \multicolumn{2}{|l|}{ Age, years, $n=37$} \\
\hline $18-27$ & $9(24.3)$ \\
\hline $28-37$ & $15(40.5)$ \\
\hline $38-47$ & $8(21.6)$ \\
\hline $48-57$ & $4(10.8)$ \\
\hline $58+$ & $1(2.7)$ \\
\hline \multicolumn{2}{|l|}{ Living arrangements, $\mathrm{n}=37$} \\
\hline I live alone & $8(21.6)$ \\
\hline I have family members living with me & $21(56.8)$ \\
\hline I live with my partner & $4(10.8)$ \\
\hline I live with one or more friends & $2(5.4)$ \\
\hline I share my house with housemates & $2(5.4)$ \\
\hline Having pets, $n=37$ & $31(83.8)$ \\
\hline \multicolumn{2}{|c|}{ Mental health service received via telehealth, $n=36$} \\
\hline Assessment & $8(21.6)$ \\
\hline Pre-treatment & $6(16.2)$ \\
\hline Individual treatment & $34(91.9)$ \\
\hline Group treatment & $19(51.4)$ \\
\hline On-request treatment & $2(5.4)$ \\
\hline \multicolumn{2}{|c|}{ Treatment duration at the start of restrictions, $n=37$} \\
\hline More than 12 months & $6(16.2)$ \\
\hline Six to 12 Months & $11(29.7)$ \\
\hline Between one and six months & $18(48.6)$ \\
\hline In assessment & $2(5.4)$ \\
\hline
\end{tabular}

\section{Copyrights}

Copyright for this article is retained by the author(s), with first publication rights granted to the journal.

This is an open-access article distributed under the terms and conditions of the Creative Commons Attribution license (http://creativecommons.org/licenses/by/4.0/). 